\title{
Le physique de la transgression : La trilogie de Mallaig
}

\section{Camille CONTRE}

Les individus ne se conformant pas à la doxa et aux lieux communs ont constamment été affublés de l'appellation «monstre ». Michel Foucault 1'explique d'ailleurs dans son cours Les anormaux : «Le monstre, en effet, contredit la loi. Il est l'infraction, et l'infraction portée à son point maximum » (37). Il définit plus loin cet être de la sorte : « le monstre est, en quelque sorte, la forme spontanée, la forme brutale, mais, par conséquent, la forme naturelle de la contre nature » (38). Transgresseur, il effraie la société par sa différence. En plus de son comportement hors nome, le monstre qui nous intéresse se définit par sa stéréotypie. Il s'agit ainsi de la forme la plus cliché de cet être : l'anormalité du corps. C'est sa différence physionomique qui le rend monstrueux. Nous analysons les anomalies, les particularités, bref l'étrangeté du corps humain dans l'œuvre paralittéraire québécoise La trilogie de Mallaig de Diane Lacombe — soit La châtelaine de Mallaig (CM), Sorcha de Mallaig (SM) et L'hermine de Mallaig $(H M)^{1}$. Nous tentons de répondre à la question : le monstre est-il la création de la société ?

De prime abord, nous traitons de la tératologie. Deux personnages sont aux prises avec des malformations et des difformités physiques, telles que présentées par Ambroise Paré dans son livre Des monstres et prodiges. Paré introduit également une autre forme de tératogène dans ces ouvrages, soit les jumeaux. Ensuite, nous analysons les maladies s'attaquant au physique des personnages de La trilogie de Mallaig. Les pathologies, dont la peste et la gangrène, répugnent les hommes en raison de la peur qu'elles inspirent. Enfin, l'étude s'arrête sur la question du corps meurtri par les coups et blessures, plus particulièrement les cicatrices.

\section{La tératologie : le physique difforme et les naissances multiples}

En premier lieu, arrêtons-nous sur la plus monstrueuse forme de transgression du corps : la tératologie. Il s'agit de la science qui traite des anomalies et des écarts physiques chez l'homme et les animaux. Les difformités et les malformations physiques ont longtemps été l'une des causes de la création d'êtres monstrueux. Toutefois, le monstre ne se situe pas qu'au niveau des difformités physionomiques. En effet, nous avons également retracé une autre forme du 
corps monstrueux : les jumeaux. Le monstre est anormal, car il s'oppose à la norme, à ce qui se conforme aux valeurs communes de la société.

Le personnage le plus représentatif de la tératologie, dans La trilogie de Mallaig, est sans aucun doute Ceit MacNèil. Apparaissant dans deux des trois romans - CM et $S M-$, cette jeune fille est mise à l'écart du reste de la communauté : elle partage les quartiers des servantes du château de Mallaig. Orpheline et bâtarde du chef du clan MacNèil, elle se voit décrite à plusieurs reprises comme étant laide et hideuse, voire «sauvage » $(C M 47)$ : «C'était une enfant malingre au visage étroit. Ses traits accusaient une légère difformité. Au premier coup d'œil, cela ne paraissait pas beaucoup, mais ses yeux étaient légèrement inégaux. Elle avait un petit menton volontaire qui détonnait dans cet ensemble empreint de fragilité »(63). Son physique accuse une légère tare. Elle cache son visage (114) afin de se soustraire aux commentaires acerbes, ce qui témoigne d'un manque d'estime de soi et d'assurance de sa part. La différence physique, face à ce qui est communément accepté, incite la jeune fille à être perçue comme un être monstrueux. L'orphelinat, la bâtardise et la laideur n'aident nullement la jeune fille à sortir de son statut de paria. Chacun de ces états, pris individuellement, est suffisant pour qu'elle connaisse une exclusion sociale. Se cacher de la communauté ne favorise pas la déconstruction de ce statut. Effectivement, cela empire puisqu'elle admet presque qu'à cause de sa différence, la société écossaise ne peut accepter qu'elle n'en devienne un membre à part entière. Ce n'est que lorsqu'elle est prise en charge par Gunelle, puis adoptée par les époux MacNèil, que la jeune fille accepte sa place à Mallaig et commence à être de plus en plus présente dans la sphère sociale. Ceit comprend donc, par l'attention parentale qu'elle reçoit de ceux qui l'adoptent, qu'elle n'a pas à se dissimuler et qu'elle doit apprendre à aimer celle qu'elle est et deviendra. Ceit n'échappe pas à ce phénomène social: enfant, elle n'arrive pas à s'apprécier car elle ne représente nullement la norme sociale. Le lecteur peut facilement s'identifier à ce personnage emblématique qui doit apprendre à surmonter la critique sociale face à sa différence physique. Paré a essayé d'expliquer pourquoi certains individus naissent de la sorte dans Des monstres et prodiges. Selon lui, ce phénomène se produirait à cause de l'étroitesse de la matrice : «Il se fait aussi des Monstres pour la détresse du corps de la matrice, comme on le voit que, lorsqu'une poire attachée à l'arbre, posée en un vaisseau étroit devant qu'elle soit accrue ne peut prendre croissance complète » (93). Ainsi, à défaut d'espace lors de la gestation, « les enfants sortent du ventre de leurs mères monstrueux et difformes » (93). Paré donne une autre explication quant à 
la difformité des enfants : «la femme étant grosse, pour s’être tenue quasi toujours assise pendant sa grossesse et les cuisses croisées [...], ou s'être bandé et trop serré le ventre, les enfants naissent courbés, bossus et contrefaits » (94). Ceit MacNèil est ainsi un protagoniste monstrueux puisqu'elle est différente, voire «contrefaite » puisqu'elle s'éloigne des conventions et devient, pour reprendre l'expression de Foucault, « contre-nature ».

Ajoutons à son physique étrange son problème de communication. La fillette souffre de surdité partielle, ce qui l'a empêchée de converser lorsqu'elle était enfant. D'ailleurs, les habitants de Mallaig «la tien [nent] pour muette » $(C M 47)$. Claude-Claire Kappler affirme que le langage est essentiel à la création identitaire d'un individu (172). Ainsi, le fait que Ceit ne puisse communiquer proprement, la renvoie à son état d'individu sauvage de même qu'à son anormalité. Elle s'isole volontairement de la communauté de Mallaig au point où seule l'intendante Anna sait que la petite parle (CM 47), ce qui a pour effet d'accentuer sa différence auprès des autres qui ne la rejette que plus facilement. Sa peur d'être jugée en fonction de son apparence physique et de sa surdité accentue l'image négative qu'elle a d'elle-même. Puisqu'elle ne parle qu'en de rares occasions, elle a un énorme retard de langage par rapport à un enfant de son âge. En raison de l'inattention des adultes à son égard, elle ne reçoit aucune aide pour surmonter son déficit d'apprentissage. Ce n'est que lorsque Gunelle s'intéresse à elle et décide de la former, que la fillette rattrape son retard et sort enfin de sa coquille. En résumé, Ceit MacNèil est monstrueuse parce qu'elle va à l'encontre de la doxa, de la norme prescrite par la société. Elle s'éloigne des conventions et devient, pour reprendre l'expression de Foucault, « contre-nature ».

Ensuite, un deuxième personnage s'offre à nous sous la bannière du tératogène. Il s'agit du frère Gabriel présent dans le second tome, SM. Son physique est anormal à cause de ses pieds qui semblent noueux (79). Lorsqu'une partie du corps - tel que les doigts, les genoux, ou dans notre cas, les pieds - est dite noueuse, cela fait référence aux nodosités ou renflements de la peau qui déforment les articulations ${ }^{2}$. Cette malformation nous incite à le classer en tant que tératogène. Paré offre une explication quant à ce type d'anormalité : « Davantage quand la mère reçoit quelque coup sur le ventre ou qu'elle tombe de haut en bas, les enfants en peuvent avoir les os rompus, déboîtés et torturés [tordus], ou recevoir autre vice, comme être boiteux, bossus et contrefaits » (97). Ainsi, il est possible d'émettre l'hypothèse que la cause des pieds noueux du moine provienne d'un coup reçu ou d'une chute subie par la mère pendant sa grossesse. La 
seconde assertion est logique et cohérente si l'on se souvient que Baltair MacNèil affirme que «c'est chose coutumière dans les Highlands » (CM 83) de maltraiter son épouse. On le sait, le monstre effraie par sa différence au point où la communauté s'en éloigne pour se dissocier de tout individu anormal. C'est d'ailleurs ce que font les moines de l'abbaye d'Iona en obligeant le frère Gabriel à aller pêcher pour le monastère, ce qui l'empêche de participer à trois des sept offices de la journée (SM 80). La vie de moine est en soi une autre forme de d'aliénation : on s'isole de toute distraction pour ne se consacrer qu'à l'Éternel. Certains religieux augmentent leur propre marginalité soit en faisant vœu de silence, soit en désirant vivre tel un reclus. Ces croyants se dissocient de tout ce qui peut nuire à leur quête spirituelle. De plus, l'abbaye d'Iona est un monastère qui se situe sur l'île du même nom. L'île entière semble retirée de la civilisation : «Où que l'on posât le regard, il n'y avait que de l'eau, des rochers et du ciel gris » (72). D’ailleurs, Sorcha « éprouv [e] un étrange sentiment de désolation » (72) lorsqu'elle arrive sur l'île avec sa mère. La région semble être un lieu désertique. Le simple fait de résider dans cette abbaye implique une isolation du reste du continent - rompue par intermittence grâce aux pèlerins - , ce qui amplifie le sentiment de rejet du frère Gabriel. La religion est normalement un élément rassembleur pour ceux qui partagent une même foi. Or, le catholicisme semble ici s'ériger en tant qu'adversaire à la vie sociale : aucun retour vers la normalité, vers la civilisation n'est possible.

Aux pieds noueux du moine solitaire s'ajoute une bouche édentée (125). N'en doutons pas, l'hygiène dentaire existait bel et bien au Moyen-Âge. Laurence Moulinier-Brogi a écrit dans «Hygiène et cosmétique de la bouche au Moyen-Âge » que «l'état de la dentition était perçu comme composante principale du visage, et partant, comme un élément d'identité et de reconnaissance » (228). Ainsi, la perte des dents est également une composante à part entière de l'identité. L'absence de dents provoque plusieurs problèmes liés à la diction. Tout comme Ceit MacNèil, la communication semble difficile pour ce moine, par ailleurs surnommé Gabriel le Bègue : «J'avais $[\ldots]$ peine à le comprendre, tant à cause de son accent que des mots latins dont il truffait chaque phrase et du bégaiement dont il était affecté » (SM 80). Forme de monstruosité et de torture personnelle, le bégaiement façonne également l'identité d'un individu. En vérité, les bègues voient, majoritairement, leur vie dominée par ce problème : «Les préjugés envers ces personnes se traduisent également en actes au travers de comportements d'exclusion et de discrimination qui enrayent la bonne insertion des jeunes dans leur milieu scolaire et limitent les 
opportunités d'emploi et de promotions des adultes. À l'adolescence, ces populations subissent un risque accru de harcèlement moral » (Bourguignon et Demoulin 181). Le personnage du frère Gabriel semble représenter le bègue moderne : il est discriminé par ses pairs et comprend que ses chances de participer pleinement à la vie ecclésiastique sont réduites en raison de son élocution. Si à cela s'ajoute des difformités physiques, le tout n'est plus homme: il se transforme en créature immonde et effrayante. Le frère Gabriel devient monstre parce que la société ne le comprend pas.

En outre, nous pourrions affirmer qu'il existe une troisième forme de monstre dans $L a$ trilogie de Mallaig. Il s'agit du cas des jumeaux Dudh et Malcolm, fils du seigneur Iain MacNèil et de son épouse Gunelle Keith. Ces deux garçons ne sont en aucun cas des êtres difformes, mais ils demeurent des tératogènes. Kappler a écrit que «[d]epuis toujours les jumeaux inquiètent. Dans l'Antiquité il arrivait qu'on les mît à mort. Notre Moyen Age les considérait comme un présage alarmant»(209). En effet, plusieurs enfants nés d'une même grossesse sont généralement associés aux animaux dans l'imaginaire collectif du Moyen-Âge et de l'Antiquité :

Le commun accouchement des femmes est un enfant ; toutefois, on voit (comme le nombre des femmes est grand) qu'elles accouchent de deux, que l'on appelle gémeaux ou bessons ; il y en a qui en accouchent de trois, quatre, cinq, six, et plus. Empédocle dit que, lorsqu'il y a une grande quantité de semence, il se fait pluralité d'enfants. Autres, comme les Stoïques, disent qu'ils s'engendrent pour ce qu'en la matrice il y a plusieurs cellules, séparations et cavités, et, quand la semence est épandue en icelles, il se fait plusieurs enfants ; toutefois cela est faux, car en la matrice de la femme il ne se trouve qu'une seule cavité, mais aux bêtes, comme chiennes, pourceaux et autres, il y a plusieurs cellules qui est cause qu'elles portent plusieurs petits. (Paré 69)

Ainsi, la mère et les enfants sont perçus comme des monstres possédant des attributs animaliers, procédant ainsi à une hybridation entre l'homme et l'animal. Pour éviter une telle catégorisation, la société essaie de dissocier les jumeaux pour qu' «un aîné et un second soient identifiés » (Scelles 76). Ce faisant, il y a une tentative de normalisation quant à la dualité identitaire des enfants monozygotes. Les jumeaux identiques doivent apprendre à se distinguer entre eux par leur personnalité. S'ils partagent les mêmes valeurs et les mêmes intérêts, ils sont la représentation d'une même et unique personne divisée. Ensemble, ils ne font qu'un ; séparés, ils ne sont rien sans l'autre. Comme le yin et le yang, l'un est la lumière, l'autre la noirceur. Leurs personnages semblent inséparables : jamais nous ne les retrouvons éloignés de l'autre. Dudh et Malcolm ne sont pas deux individus, mais bien une seule et unique personne ${ }^{3}$. La société semble 
ici n'apporter aucune importance quant à la double naissance, au titre d'aînesse. Ces garçons ne peuvent être dissociés puisqu'ils ne sont et ne font qu'un. Dudh et Malcolm sont monstrueux en raison de leur dualité identitaire.

D'autre part, Olivier Roux affirme, dans Monstres: Une histoire générale de la tératologie des origines à nos jours, qu'une femme donnant naissance à plus d'un seul enfant lors d'une même grossesse peut «trahi [r] à l'encontre de la mère un comportement adultérin » (190). Le cas de l'adultère est d'autant plus frappant si les enfants ne sont pas identiques. Nous trouvons d'ailleurs quelques exemples assez frappants de jumeaux n'ayant pas le même géniteur dans la mythologie grecque, notamment avec le récit des jumeaux Héraclès et Iphiclès ${ }^{4}$. Chaque enfant aurait hérité des traits physionomiques de leur père respectif, confirmant ainsi l'hypothèse que l'épouse aurait eu un amant. De cette manière, nous pouvons conclure que la présence des jumeaux est doublement monstrueuse : les enfants issus de ces grossesses sont perçus tels des animaux et la mère semble avoir des relations extra conjugales. Cependant, dans $S M$, rien n'indique que Gunelle Keith ait partagé le lit d'un autre homme puisqu'aucun détail physique, outre la taille de Dudh et de Malcolm (379), n'est mentionné. Roux affirme que certains jumeaux sont perçus comme des êtres exceptionnels (190), ce qui est le cas des jumeaux MacNèil. Les naissances gémellaires se présentent dans le second tome en tant que monstres dissimulant un filon peu connu de la tératologie. En d'autres mots, Ceit MacNèil et le frère Gabriel sont deux tératogènes dont la physionomie effraie parce qu'elle est hors norme. Dudh et Malcolm sont également des êtres monstrueux puisqu'ils sont jumeaux. Ces naissances multiples rappellent celles des animaux, ce qui en confirme l'étrangeté. . L'anormalité, la «contre-nature », voilà ce qu'est le monstre.

\section{Les maladies : la peste et la gangrène}

En second lieu, les maladies sont également vectrices de la création d'un monstre social. Les personnes atteintes d'une infection risquent d'être rejetées et mises à l'écart par la société qui ne souhaite pas être en leur présence de peur d'être contaminée à son tour. Il existe de multiples infections pouvant modifier la physionomie d'une personne. Par exemple, la petite vérole laisse, parfois, des cicatrices sur le corps qui peuvent grandement affecter l'apparence. En ce qui concerne La trilogie de Mallaig, nous trouvons deux maladies qui transforment le physique d'une horrible manière : la «mort noire » et la gangrène. 
La peste constitue la première infection. Cette terrible maladie a fait des ravages au Moyen-Âge. Les victimes sont d'abord prises de fièvre, de frissons et de vertiges. Puis, survient le bubon, excroissance horrible des glandes lymphatiques. La mort suit d'environ une semaine l'apparition des abcès. Dans le premier tome $C M$, le châtelain Iain MacNèil est confronté à ce terrible fléau. En chemin pour aller rencontrer le roi d'Écosse, Iain et sa troupe se retrouvent dépourvus face à la désolation des routes. Un cavalier vient à leur rencontre et demande l'assistance du révérend. Cependant, il interdit l'accès à la ville à tous les autres voyageurs. C'est après le retour du religieux, resté une nuit auprès des habitants du château de Doune, que la troupe comprit de quoi il retournait :

Le [révérend Henriot] avait l'air abattu et vidé de toute son énergie. Il se laissa glisser sur le sol, se signa en contemplant les murs du château et se mit en prière quelques instants. Interdits, les hommes l'observèrent en silence, une vague appréhension leur serrant le cœur. Quand le révérend leur adressa finalement la parole, ce fut pour faire le récit désolant de ce qu'il avait vécu dans les murs.

«Mon seigneur, dit-il en regardant Iain, c'est la peste. Au château, sept personnes en sont mortes la semaine dernière, plus leur prêtre avant-hier et trois autres hommes cette nuit. [...] Ils ont contracté la maladie dans les prisons du roi où le fléau fait ravages en ce moment. [...]». (459-460)

De ce récit navrant, ils concluent que la maladie expliquait l'absence de toute âme qui vive sur leur route. Le signe de la croix que le révérend fait de ses mains, alors qu'il regarde le château, est le même que celui qu'il aurait fait au moment de mettre un cercueil en terre. Le château devient le tombeau des pauvres âmes qui y circulent. Le révérend laisse croire qu'il n'y a plus d'espoir pour eux. De cette manière, Iain et ses chevaliers entreprennent une lente descente aux Enfers. La mort pourrait les happer à tout instant s'ils attrapaient le virus de la peste. Cette maladie incite la peur :

J'avais tant lu à Orléans sur ce terrible fléau qu'on accusait d'avoir décimé la moitié de la population de France en moins de cent ans ! Comme tous, je redoutais la peste qui allait et venait à travers toute l'Europe et qui ne semblait jamais se rassasier de victimes. Elle prenait ici cent âmes, là vingt, au gré de sa fantaisie, dans les bourgs comme dans les villes. (464)

Personne ne semble pouvoir échapper à cette maladie, personnifiée dans le récit. La peste devient donc, à l'image de la mort faucheuse, une entité parcourant le vieux continent à la recherche de sa prochaine victime. Ne sachant jamais où ni qui elle frappera, cette maladie a engendré un sentiment de peur panique chez tous les Européens, peu importe leur caste. D’ailleurs, même « [l]e roi ne recevait plus, ne convoquait plus, n'emprisonnait plus et mettait tous ses gens 
en demeure de combattre, avec leur pitoyable armes » (464) dans son château afin d'éviter toute contamination avec la population. D'autre part, le corps médical du Moyen-Âge reçoit de nombreuses critiques, car les médecins sont «[d]épourvus de moyens efficaces sur le plan thérapeutique » (Fabre 83). Par exemple, les habitants de Doune ont cru bon d' «imbib[er] tous [1]es vêtements » $(C M 460)$ du révérend de vinaigre, mais ce dernier « doute de [1']efficacité » (460) de cette technique. Dans le but d'enrayer la maladie, « [Iain MacNèil] avait participé [...] à l'incendie des maisons des victimes et à la protection des puits que l'on interdisait aux pestiférés, de peur qu'ils n'en contaminent l'eau » (464). La décontamination et la destruction des biens matériels ayant été en contact avec le bacille de la mort noire semble justifiées : il s'agit d'une tentative du retour à l'ordre, à la normale. La communauté tente de purifier la ville en la réduisant en cendre pour recommencer une nouvelle vie. Cette maladie était doublement effrayante : non seulement une grande partie de la population mourait dans d'atroces souffrances, mais on ne connaissait aucun remède. La peur propage davantage la maladie que le virus. Fabre le confirme : « la peur prédisposerait à la contamination : le 'levain de la frayeur' — autrement dit «ce qui suscite la peur» — serait même indispensable, prétendent certains tenants de l'aérisme, à la survenue de l'infection » (87).

Au Moyen-Âge, si le rationnel ne pouvait pas fournir d'explications, alors on se tournait vers l'irrationnel: Dieu. Ainsi, pour certains, la peste était le reflet de la colère du Seigneur déversée sur Terre :

«C'est l'air du midi qui amène cette maladie... et c'est une punition du ciel. »

Se remettant à genoux, [le révérend Henriot] invita la compagnie à en faire autant :

«Prions, mes frères. Dieu nous écoutera si nous sommes repentants et, dans sa grande sagesse, Il nous épargnera. » (CM 460)

Paré consacre un chapitre au sujet des maladies d'origine divine. Il affirme que certaines maladies sont envoyées par Dieu et qu'aucun remède traditionnel ne peut les guérir (158). La peste ne choisit pas un individu en particulier, tous y sont d'éventuelles victimes. Le voile de la mort étend sa domination en semant la peur sur son passage. Bref, le pestiféré est évité autant que possible. On le place d'ailleurs en quarantaine pour empêcher la propagation du virus. L'horreur de cette maladie - la transformation du corps et la mort inévitable qui s'ensuit attise la peur des individus. Le physique, devenu monstrueux, semble être l'œuvre du Tout Puissant. 
La seconde pathologie affectant le physique est la gangrène. Il s'agit d'une maladie qui apparaît sur une plaie qui n'a pas été correctement soignée. Dans $S M$, le seigneur Iain MacNèil souffre de cette infection. Tout à commencer par une blessure au genou qu'il s'est faite lors d'un combat (355). Avec le temps, la blessure a empiré au point d'affecter sa mobilité : il a de la difficulté à marcher, à monter à cheval (355) et à se tenir en équilibre lorsqu'il est debout (386). La plaie sur son genou ne semble pas guérir (356). La description de Sorcha Lennox reflète son dégoût tout en décrivant la progression de l'infection : «la jambe était enflée à un point tel que je ne distinguais plus le pli de flexion. Autour de la plaie, de la cuisse au mollet, la peau était crépitante et aussi brunâtre que le liquide qui suintait du genou » (408). Le corps d'Iain MacNèil se transforme pour devenir monstrueux et effrayant. La vue de la jambe de cet homme en indispose certains au point qu'ils refusent de venir rendre visite au malade, comme c'est le cas de Ceit MacNèil (408). De plus, un autre sens entre en jeu, soit celui de l'olfaction. Le dégoût ressort d'autant plus lorsque l'énonciation indique qu' « une odeur nauséabonde » (385) s'en dégage ou que la blessure est une «immondice puante» (408). Le médecin des MacNèil ainsi que la guérisseuse Sorcha Lennox affirment tous deux que, pour sauver la vie d'Iain, il faut procéder à l'amputation de sa jambe. Cependant, ce dernier refuse d'être opéré puisqu'il veut être enterré en un seul morceau (408). Sans amputation, l'infection se propage rapidement et mène inévitablement à la mort. La gangrène est monstrueuse en raison de l'état dans lequel se trouve le patient une fois que la putréfaction de la chair empêche toute mobilité. En effet, le malade se trouve dorénavant dans un état végétatif : « le chef MacNèil s'amenuisait à l'abri des courtines de son lit qu'il ne quittait plus»(401). Anne-Laure Boch affirme que «[1]'état végétatif, c'est le handicap le plus extrême qu'on puisse imaginer, la pointe avancée de la dépendance » (76-77). Restreint à un espace confiné, le malade perd son autonomie, son autorité, voire son identité. Il n'est plus. Il ne fait qu'attendre patiemment que la mort vienne le délivrer de tous ses maux. La gangrène est ainsi une infection monstrueuse : elle s'attaque au corps du blessé pour le rendre presque méconnaissable et l'amène à sa propre mort.

Par ailleurs, afin de se soulager de la souffrance occasionnée par sa blessure, le chef du clan se saoule (SM 388). L'ivresse, variante de l'automutilation, détruit le corps à petit feu. La consommation excessive d'alcool peut, à long terme, aboutir à une cirrhose et pis encore, au décès. Entre la vie et la mort, le whiskey apparaît à Iain MacNèil comme une véritable délivrance. Cette eau-de-vie devient peu à peu une nécessité, au point où le chef du clan ne peut 
s'en passer. N'y verrait-on pas ici une représentation du malade moderne dépendant de la morphine qui lui permet d'oublier la douleur? La société pointe du doigt celui qui se transforme en monstre en raison de ses excès. Il s'isole et diffère des autres en préférant taire ses souffrances par la bouteille. Bien qu'il souffre en silence, le comportement d'Iain est beaucoup plus agressif que normalement. Il ne parle presque plus, il crie (384-386, 398). L'alcoolique s'automarginalise en s'isolant de tout ce qui n'est pas associé à la boisson. Il est le seul responsable de son intoxication. Iain boit par nécessité ; il sait que sa mort est inévitable, mais ne peut rien y changer. Sans morphine au Moyen-Âge, l'analgésique le plus effectif restait l'alcool. En résumé, la peste et la gangrène sont deux terribles maladies présentes dans La trilogie de Mallaig. La mort guette celui qui en souffre. Le corps s'en trouve déformé et devient monstrueux aux yeux de tous. La mise en quarantaine du pestiféré et la perte de l'usage d'un membre à la suite d'une infection effraient le commun des mortels.

\section{Le corps meurtri}

Le monstre physique apparaît aussi lorsque le corps est blessé. Précédemment, nous avons mentionné que certaines maladies affectent l'apparence d'un individu. Ici, il est question des blessures qui transforment la physionomie en monstruosité. Le corps meurtri rend compte de la violence sociale en raison des idéologies menant à la mutilation de la peau. Ce monstre physionomique semble se construire davantage lors de conflits où le désir de possession des hommes est à son comble. Chaque blessure renvoie à la violence qui a été nécessaire afin d'acquérir l'objet de convoitise.

Dans $H M$, la physionomie de Baltair MacNèil souligne l'idée que les desseins de possession des hommes jouent un rôle par rapport au statut de monstre. Cet homme faisait partie de la troupe d'Alexandre Stuart et était considéré comme un cateran en Écosse, c'est-à-dire un brigand. Maurice Cusson décrit avec justice la violence perpétrée par ces hommes en affirmant que «des chevaliers brigands se livraient à des exactions, attaquaient les abbayes, les dépouillaient, tuaient des moines, mettaient le feu et emportaient argent, calices, livres précieux, chevaux » (85). On comprend clairement que ce métier est extrêmement dangereux : ils pouvaient se blesser lors des assauts, mais également être condamnés à mort par les autorités s'ils étaient arrêtés. Alors que Baltair pillait les maisons du bourg d'Elgin, l'une des résidences s'est effondrée sur lui. Il s'en sort miraculeusement en n'ayant pour blessure qu'une cheville 
fracturée. Dès lors, il lui est difficile de se déplacer et de se défendre contre les multiples ennemis qu'il s'est fait au cours des raids auxquels il a participé (HM 71). Il ne peut ni courir ni mettre son poids sur son pied blessé, ce qui le rend dorénavant vulnérable. Bien qu'il ne s'agisse pas d'une blessure fatale, elle affecte tout de même le physique de l'individu pendant une certaine période. L'articulation fêlée (66) fait incontestablement souffrir le blessé. Tel un infirme, il ne peut plus utiliser sa jambe convenablement, au risque d'aggraver la fracture. Il se voit donc contraint d'immobiliser sa cheville à des fins de guérison (75). D'autre part, les cicatrices recouvrent son corps (255) et accentuent ainsi la vraisemblance de son passé de mercenaire. Son corps est ainsi le rappel constant de son passé tumultueux. Le corps de Baltair MacNèil est un véritable livre d'histoire : les cicatrices, tel un tatouage, racontent le récit de sa vie. Monstruosité physique, la mutilation de sa peau rend compte des idéologies amorales auxquelles il se conformait autrefois : incendier les abbayes et les villages, piller les monastères et les maisons, violer les femmes et se livrer à la débauche dans les lupanars.

Un second personnage est également aux prises avec un corps meurtri. Il s'agit du petitfils de Baltair MacNèil, soit Baltair le Jeune. Son père l'a flagellé. Ce dernier avait décidé de le punir parce que Baltair l'avait espionné. Le fouet était utilisé pour châtier un individu au MoyenÂge. La société médiévale dépeinte dans ce roman met en œuvre une certaine violence. Le fouet n'est pas que l'unique moyen de punition : ils avaient également recours aux coups de bâton et de pelle (50). Le jeune homme devient lui-même monstrueux lorsqu'il demande à son père d'aller jusqu'au bout de sa punition, comme si ce qu'il avait déjà enduré n'était pas suffisant : « [Iain MacNèil] s'appliqua à fouetter le plus rapidement et le moins fort possible afin d'atténuer le supplice de son fils. Il tenta même d'abréger la punition en s'arrêtant au cinquième coup mais, à son grand désarroi, il fut rappelé à l'ordre par le garçon qui se mordait les lèvres pour ne pas crier » (53). Ayant eu un comportement hors norme - car qui n'aurait pas demandé grâce à sa place ? - , il se voit maintenant marqué à jamais pour son insolence enfantine. Baltair n’est en aucun cas un personnage à tendance masochiste. En réalité, il désire prouver qu'il fait enfin partie du monde des adultes (50-51). C'est en acceptant la totalité de sa sentence qu'il parvient à devenir un homme et à être perçu comme tel. De telles punitions laissent des traces. Ainsi, le désir d'ascension du jeune homme lui a permis de devenir un monstre à deux niveaux : un monstre physique, puisque son corps sera marqué à jamais, et un monstre moral en raison de son acharnement à poursuivre son châtiment jusqu'au bout. 
Il est blessé à une seconde reprise dans le roman. Cette fois-ci, son assaillant n'est nul autre que Sorcha Lennox. La jeune fille était sur le point de se faire agresser par trois vandales d'Édimbourg lorsque Baltair le Jeune et son compatriote Colm viennent la sauver. Toutefois, croyant être à nouveau attaquée, elle a poignardé le premier à l'épaule. Cette blessure a de plus graves conséquences que les coups de fouet. La plaie étant profonde, la guérison nécessite plus de temps et davantage de soin pour un rétablissement complet. D'ailleurs, Baltair ne semble guère apprécier son bandage qui, selon lui, l’empêche de se défendre : «Soudain, mon blessé prit conscience que l'écharpe, que je lui avais confectionnée tout en bavardant, immobilisait complètement son bras droit. [...] Mais, Sorcha, tu ne peux pas me ficeler comme ça ! Comment ferai-je pour me battre si je n'ai plus l'usage de ma droite ?» (354). Le fait que son bras droit soit en écharpe l'empêche de se défendre. Tout comme son grand-père, il devient vulnérable le temps de sa convalescence. Cependant, ayant à se battre, il enlève son pansement et sa blessure se met inévitablement à saigner à plusieurs reprises, retardant ainsi la guérison de son bras. Sorcha prévient même Baltair de l'importance de ses traitements : «Tant que tu saignes, la plaie n'est pas guérie et peut s'infecter. Si elle s'infecte, elle ne cicatrisera pas. Quand veux-tu recommencer à utiliser ton bras ? Ce mois-ci ou l'autre ou l'autre après ? » (371) Les risques d'infection devraient motiver Baltair le Jeune à poursuivre son traitement auprès de Sorcha Lennox, surtout que la blessure au genou de son son père Iain MacNèil le tue à petit feu. Malheureusement, puisque Baltair a préféré se battre malgré les indications contraires de la guérisseuse, il garde des séquelles de sa blessure :

- Sorcha, mon père vient de le dire : c'est mon bras qui ne se rétablit pas, me répondit Baltair, tout bas. La plaie est refermée et presque entièrement cicatrisée, mais je ne peux plus lever le bras plus haut que le coude. Je suis à l'entraînement dans cette chambre depuis deux jours devant lui. Je ne peux manier ni claymore ni épée correctement... Je crois que tu as sectionné un nerf ou un tendon avec ton coup de couteau. » (397)

En d'autres mots, Baltair le Jeune a aggravé sa blessure en décidant de défendre sa vie et celle de sa compagne lors de leur voyage de retour. Contrairement aux cicatrices qui peuvent être dissimulées sous une chemise, il ne pourra point cacher sa vulnérabilité à ses ennemis. En tant que futur chef du clan, il se doit d'être en mesure de se battre et de défendre ses terres. Il est ainsi devenu une cible facile pour ses assaillants. Baltair apparaît dès lors, tel un soldat amputé, comme un vétéran nostalgique rêvant aux combats qu'il a menés sachant fort bien qu'il ne pourra plus faire partie des troupes. Le clan de Mallaig pourrait décider de nommer un autre chef 
puisqu'il le considère comme inapte à prendre les armes. D'ailleurs, Iain MacNèil craint que son fils ne puisse lui succéder à cause de son bras invalide. Baltair le Jeune est un homme meurtri par le fouet et par le poignard d'une femme. La correction que son père lui a admonestée l'a transformé en monstre à cause de son propre acharnement. Sa blessure à l'épaule est, quant à elle, la transformation de l'homme en pleine santé à l'infirme. Les coups de fouets consolident la réputation de Baltair en tant qu'homme, tandis que l'invalidité de son bras la détruit.

Somme toute, le corps meurtri par les coups et blessures incite le monstre corporel à se développer. Par leurs cicatrices de guerre ou leur peau tannée par le cuir, Baltair MacNèil et Baltair le Jeune ont perverti leur corps en laissant l'horreur de leur passé les marquer de la sorte. Tels un livre d'histoire ou encore un tatouage, chaque cicatrice raconte et commémore ceux qu'ils étaient naguère. La fracture de la cheville ainsi que la mutilation de l'épaule font de ces deux hommes des individus infirmes et vulnérables. Seul Baltair MacNèil, grand-père de Baltair le Jeune, a pu guérir complètement. Ainsi, sa transformation en tant que telle n'a été que temporaire.

Pour conclure, le monstre se définit par son anormalité, par sa différence. La tératologie est vectrice de monstres à cause de sa foudroyante étrangeté. Tel un monstre exposé dans une foire, le tératogène ne peut éviter les critiques acerbes. Il s'auto-marginalise en préférant s'isoler plutôt que d'intégrer la communauté puisqu'il n'aime pas son apparence. Les jumeaux sont hors norme car ils représentent une seule et unique personne. Leur identité est étrange et porte à confusion, car ils ne semblent pas être en mesure d'être unique sans la présence de l'autre. Le portrait tératologique des personnages s'insère dans La trilogie de Mallaig comme une vaste critique de la société moderne et de son mode de pensée. Les pathologies affectent le physique en procédant à une horrible transformation corporelle qui isole le malade du reste de la communauté parce qu'il est la menace du statu quo. Ne devient prodige que celui dont le corps a subi un changement flagrant, voire une mutation. Le corps meurtri rend compte des désirs de possession de l'homme. Les blessures servent de livres d'histoires puisqu'elles gravent à jamais dans la chair le motif du conflit.

L'individu normal se transforme en créature immonde, sortie tout droit de nos pires cauchemars. Mais ces peurs n'émergent pas sans point d'origine : la société y a contribué. La différence physionomique et la laideur d'un individu ont de tout temps constitué une raison pour porter un jugement de valeur. La supériorité, liée à la perfection, constitue la véritable raison de 
l'apparition d'horribles créatures. Se conformer à la norme, voilà le mandat doxique de la société. Marc Angenot affirme que « [1]a plus grande et plus intense passion idéologique étant la haine avec sa diabolisation de 'monstres moraux'; on peut admettre qu'il existe une gnoséologie, une logique cognitive de la haine » (390). Haine qui se transmute de génération en génération en raison de la peur de la communauté face à l'étrangeté, à l'inconnu. Ainsi, ne seraitce pas plutôt la société qui est monstrueuse?

\section{Bibliographie}

Angenot, Marc. Dialogues de sourds : Traité de rhétorique antilogique. Paris : Arthème Fayard (Mille et une nuits), 2008.

Boch, Anne-Laure, «La médecine technoscientifique dépassée par ses monstres ». Monstres contemporains: Médecine, société et psychanalyse. Dir. Céline Masson et Catherine Desprats-Péquignot. Paris : In press (Pandora), 2015 : 67-99.

Bourguignon, David et Stéphanie Demoulin. «Bégaiement et stigma social ». Les bégaiements de l'adulte. Dir. Bernadette Piérart. Wavre: Mardaga (PSY-Évalutation, mesure, diagnostic), $2011: 167-188$.

Cusson, Maurice. Les homicides : Criminologie de la violence et de la non-violence. Montréal : Hurtubise, 2015.

Fabre, Gérard. «Les savoirs sur la contagion: la peste et l'institution de la quarantaine ». Culture, institution et savoir. Dir. André Turmel. Québec : PU de Laval (Culture française d'Amérique), 1997 : 83-106.

Foucault, Michel. Les anormaux. Cours au collège de France. 1974-1975. Paris : Gallimard/Seuil (Hautes Études), 1999.

Guirand, Félix et Joël Schmidt. Mythes et mythologies. Paris : Larousse (in extenso), 2008.

Kappler, Claude-Claire. Le monstre : Le pouvoir de l'imposture. Paris : PUF, 1980.

---. Monstres, démons et merveilles à la fin du Moyen-Âge. Paris : Payot, 1999.

Lacombe, Diane. La châtelaine de Mallaig. Montréal : VLB, 2002.

--- . L'hermine de Mallaig. Montréal : VLB, 2005.

---. Sorcha de Mallaig. Montréal : VLB, 2004.

Moreau, Martin. Dictionnaire du vocabulaire savant de la langue française. Paris : Publibook, 2013. 
Moulinier-Brogi, Laurence. «Hygiène et cosmétique de la bouche au Moyen-Âge ». Dents, dentistes et art dentaire: Histoire, pratiques et représentations Antiquité, Moyen-Âge, Ancien Régime. Dir. Frank Collard et Évelyne Samama. Paris : Harmattan, 2012 : 221-239. Paré, Ambroise. Des monstres et prodiges. Paris : Gallimard (Folio classique), 2015.

Roux, Olivier. Monstres : Une histoire générale de la tératologie des origines à nos jours. Paris : CNRS, 2008.

Scelles, Régine. «Réflexions autour du double fraternel ». Imaginaire et inconscient 14.2 (2004) : 71-82.

\section{Notes}

${ }^{1}$ Nous citerons, dans le corps du texte, par les abréviations suivantes les trois romans de la trilogie de Mallaig : CM pour La châtelaine de Mallaig, SM pour Sorcha de Mallaig ainsi que HM pour L'hermine de Mallaig.

${ }^{2}$ Martin Moreau. Dictionnaire du vocabulaire savant de la langue française. Paris : Publibook, 2013 , p. 482.

${ }^{3}$ Michel Tournier avait d'ailleurs fait de même avec les jumeaux Jean et Paul - plus connus sous le nom de JeanPaul - dans son livre Les Météores.

${ }^{4}$ Pour plus d'informations les concernant, voir Félix Guirand et Joël Schmidt. Mythes et mythologies. Paris : Larousse (in extenso), 2008.

${ }^{5}$ Rappelons qu'en Écosse médiévale, il était admis que l'homme descendait d'Adam et Ève. Ainsi tout lien rapprochant l'homme à l'animal était mal perçu par la société. 